\title{
Peripheral-type primitive neuroectodermal tumor of the ovary with EWSR1-FLI1 fusion transcript: a case report and brief review of literature
}

\author{
Corina GRIGORIU ${ }^{1,2)}$, DANA CRISTINA TERZEA ${ }^{3)}$, ANTONIA CARMEN LISIEVICI ${ }^{4)}$, \\ TiBeriu Augustin GEORGESCU ${ }^{4,5}$ ), ANDREEA ELENA CONSTANTIN ${ }^{1}$,

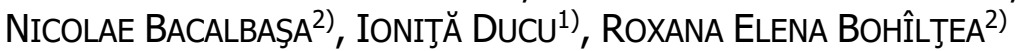 \\ 1) Department of Obstetrics and Gynecology, University Emergency Hospital, Bucharest, Romania \\ 2) Discipline of Obstetrics-Gynecology, Carol Davila University of Medicine and Pharmacy, Bucharest, \\ Romania \\ 3) Department of Pathology, Onco Team Diagnostic, Bucharest, Romania \\ 4) Department of Pathology, Carol Davila University of Medicine and Pharmacy, Bucharest, Romania \\ 5) Department of Pathology, Alessandrescu-Rusescu National Institute for Mother and Child Health, \\ Bucharest, Romania
}

\begin{abstract}
Primitive neuroectodermal tumors (PNETs) of the ovary are extremely rare tumors composed of undifferentiated small cells with round nuclei and scant cytoplasm. They are rare in general and extremely rare in the female gynecological tract, where they most commonly affect the ovary, followed by the uterine corpus. The most common presenting symptoms are abdominal pain, bloating and the presence of a pelvic mass. Diagnosis mainly relies on immunohistochemical and fluorescence in situ hybridization (FISH). Due to the rarity of these tumors, there are no standard therapeutic guidelines and treatment consists of surgery, various chemotherapy regimens and/or radiotherapy. In this article, we report the case of a 30-year-old female with peripheral-type PNET (pPNET) of the ovary featuring Ewing sarcoma breakpoint region $1-$ Friend leukemia integration 1 (EWSR1-FL/1) fusion transcript, confirmed by next-generation sequencing (NGS).
\end{abstract}

Keywords: primitive neuroectodermal tumor, EWSR1-FLI1, next-generation sequencing.

\section{a Introduction}

The term 'primitive neuroectodermal tumor' (PNET) was first introduced by Hart \& Earle, in 1973, to describe a group of small round cell tumors that appeared to be derived from fetal neuroectodermal cells with variable degrees of neuroectodermal differentiation [1]. The most frequently affected sites are the paravertebral region and the chest wall. They can be subdivided into central-type PNETs (cPNETs) and peripheral-type PNETs (pPNETs). In the last four decades, primary ovarian PNETs have been reported in less than 20 scientific articles. Ovarian PNETs usually arise in young adult females, especially before the age of 40 [2]. Common presenting complaints of the patients are the presence of a mass in one of the flanks, acute pain, weight loss or hirsutism [3-5]. Primary ovarian PNETs can be divided into cPNETs and pPNETs. The former category has a histological aspect similar to primitive tumors arising in the central nervous system, and consequently is considered to arise in nervous tissue present in ovarian teratomas. This category also has a particular immunohistochemical (IHC) profile, characterized by absent immunoreactivity for cluster of differentiation 99 (CD99) [5, 6]. pPNETs are even less commonly encountered in comparison to cPNETs, and they are characterized by the presence of a diffuse proliferation of small round blue cells forming rosettes [7]. pPNETs also feature diffuse immunoreactivity for CD99 [3]. PNETs are additionally characterized by the presence of classical translocations Ewing sarcoma breakpoint region 1-Friend leukemia integration 1 (EWSR1-FLII), although other partner fusions for EWSR1 have been reported [8-10].

\section{Aim}

In this case report, we aim to describe the obstetric and oncological outcome of a young patient with an International Federation of Gynecology and Obstetrics/ Fédération Internationale de Gynécologie et d'Obstétrique (FIGO) stage IIB pPNET of the ovary, initially diagnosed as an adult-type granulosa cell tumor and successfully treated with combined radiation therapy and chemotherapy.

\section{ㅁ Case presentation}

A 30-year-old woman presented in May 2021 to the Department of Obstetrics and Gynecology, University Emergency Hospital, Bucharest, Romania, for a gynecological consultation, accusing low abdominal pain and irregular vaginal bleeding. Upon genital examination, a hard, painful mass was palpable in the right parauterine area. Ultrasound (US) examination revealed a tumoral mass imprinting the posterior uterine wall, with complex echogenicity, associated with a small hemorrhagic collection at the bottom of the Douglas pouch. Serum tumor markers cancer

This is an open-access article distributed under the terms of a Creative Commons Attribution-NonCommercial-ShareAlike 4.0 International Public License, which permits unrestricted use, adaptation, distribution and reproduction in any medium, non-commercially, provided the new creations are licensed under identical terms as the original work and the original work is properly cited. 
antigen 125 (CA125), human epididymis protein 4 (HE4), carcinoembryonic antigen (CEA) and carbohydrate antigen 19-9 (CA19-9) were negative. Native and intravenous contrast pelvic magnetic resonance imaging (MRI) revealed a process replacement process of approximately 110/90/90 mm located in the pelvis, at the bottom of Douglas pouch, with superior development in the median and right paramedian area, to the level of the iliac crest, which imprints the posterior wall of the uterus, imprints and deflects the appendages bilaterally, with demarcation edge present, comes in posterior contact with the rectum and dislocates the intestinal loops. The described mass had inhomogeneous $\mathrm{T} 1$ and $\mathrm{T} 2$ signal, with areas of $\mathrm{T} 2$ isosignal and T1 hypersignal within and minimal, non-gadolinophilic, diffusion restriction. Three days after the MRI, the patient complains of intense abdominal pain. A new US revealed a large hemorrhagic collection in Douglas pouch. The patient was referred for surgical treatment.

An emergency laparotomy evidenced massive hemoperitoneum and a $11 \mathrm{~cm}$ dilacerated tumor imprinting the posterior wall and the right lateral border of the uterus (Figure 1). The tumor was adherent to the sigmoid. A clear cleavage plane allowed the surgeon to perform a dissection of the uterine mass from the underlying myometrium. Postoperative course was smooth, and the patient was discharged. The tumorectomy specimen, along with salpingectomy and partial omentectomy were sent to the Department of Pathology for histopathological examination.

Gross inspection of the tumor revealed a soft, fleshy mass, with gray-tan cut surface, featuring extensive areas of hemorrhage and necrosis. No teratomatous elements were noted. Intraoperative frozen-section examination suggested a torsioned granulosa cell tumor. Histopathological examination revealed a diffusely infiltrative, poorly differentiated proliferation, composed of small cells with hyperchromatic, round nuclei and finely granular cytoplasm, arranged in solid or insular patterns, with occasional rosette formation, increased mitotic activity and extensive areas of necrosis (Figures 2 and 3). Subsequent IHC testing revealed diffuse positivity for vimentin, CD99 and FLI1. Synaptophysin and cluster of differentiation 56 (CD56) were also positive in relatively frequent tumor cells. Glial fibrillary acidic protein (GFAP) was mostly negative, with very few isolated positive tumor cells (Figures 4-9). Pancytokeratin (CK) AE1/AE3, epithelial membrane antigen

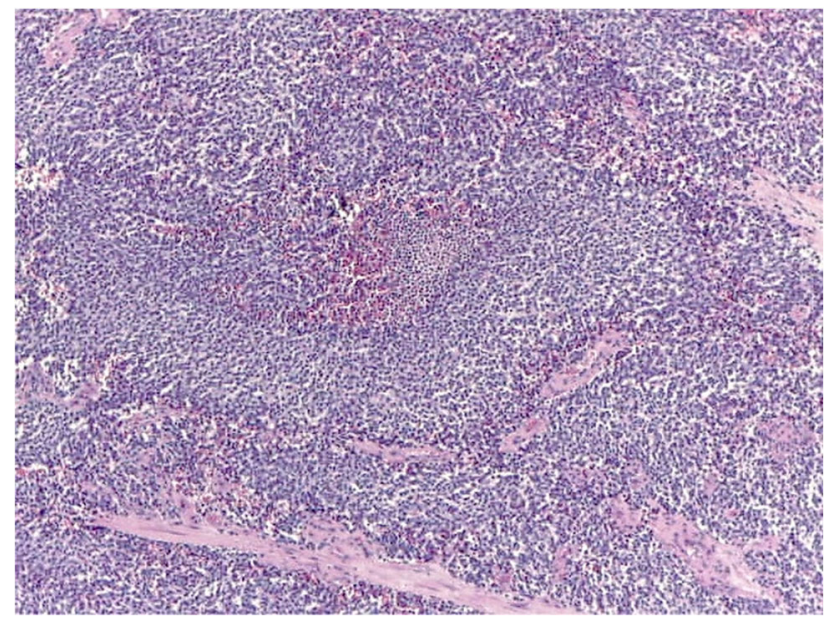

Figure 2 - The tumor was composed of a diffuse proliferation of small blue cells (HE staining, 100×). HE: Hematoxylin-Eosin.
(EMA), inhibin, Wilms' tumor 1 (WT1) and desmin were completely negative within all tumor cells. Ki67 proliferative index was approximately $60 \%$. In conclusion, the cytomorphological aspect, correlated with the IHC panel was suggestive for a malignant neuroectodermal tumor, not otherwise specified, FIGO stage IIB. The pathological report referred the case to molecular testing for EWSR 1 rearrangement. A total of 58 sarcoma related fusions were simultaneously tested using Ion AmpliSeq Ribonucleic Acid (RNA) Fusion Panel (Thermo Fisher Scientific), including, but not limited to: activating transcription factor 1 (ATF1)-EWSR1, EWSR1-ATF1, EWSR1-cyclic adenosine monophosphate (cAMP)responsive element-binding protein 1 (CREB1), EWSR1deoxyribonucleic acid (DNA) damage inducible transcript 3 (DDIT3), EWSR1-E twenty-six (ETS) transcription factor ERG $(E R G), E W S R$ l-ETS variant transcription factor 1 (ETVI), EWSR1-ETS variant transcription factor 4 (ETV4), EWSR 1-FEV transcription factor, ETS family member $(F E V), E W S R 1-F L I 1, E W S R 1-$ nuclear factor of activated T-cells 2 (NFATC2), EWSR1-nuclear receptor subfamily 4 group A member 3 (NR4A3), EWSR1-POZ/ $\mathrm{BTB}$ and AT-hook-containing zinc finger 1 (PATZ1), EWSR 1-pre-B-cell leukemia transcription factor $1(P B X 1)$, EWSR1-switch/sucrose non-fermentable (SWI/SNF)-related matrix-associated actin-dependent (SMARCA5), EWSR1Sp3 transcription factor (SP3), EWSR1-YY1 transcription factor (YY1), EWSR1-zinc finger protein 384 (ZNF384), EWSR1-zinc finger protein 444 (ZNF444). Sequencing was carried out using the Next-Generation Sequencing (NGS) Platform Ion proton (Thermo Fisher Scientific). The assayed sample expressed the EWSR1-FLII transcript. Based on these findings, the final diagnosis was pPNET.

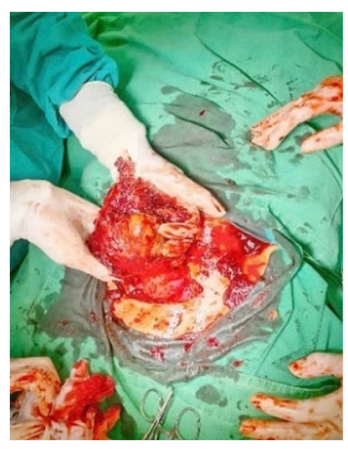

Figure 1 - Intraoperative gross aspect of the excised tumor.

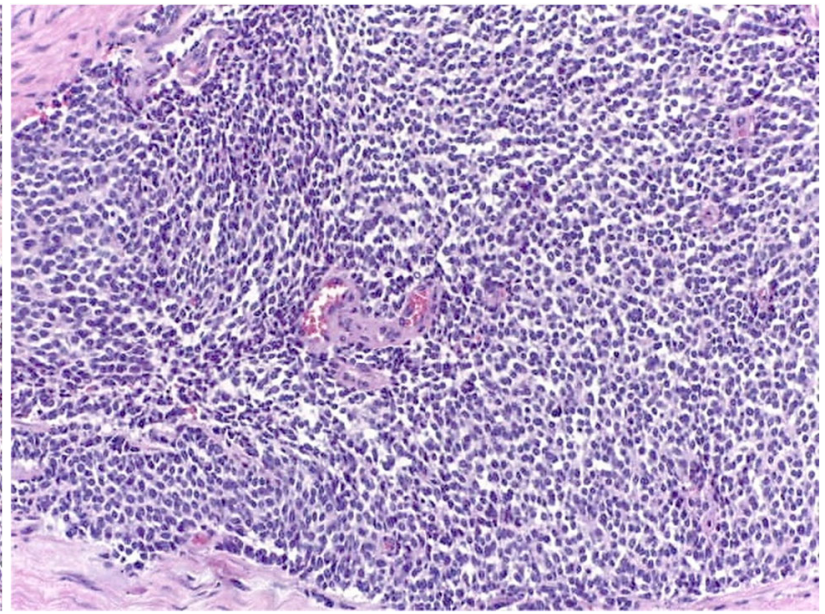

Figure 3 - The tumor showed focal rosette formation (HE staining, 200 $\times$ ). 


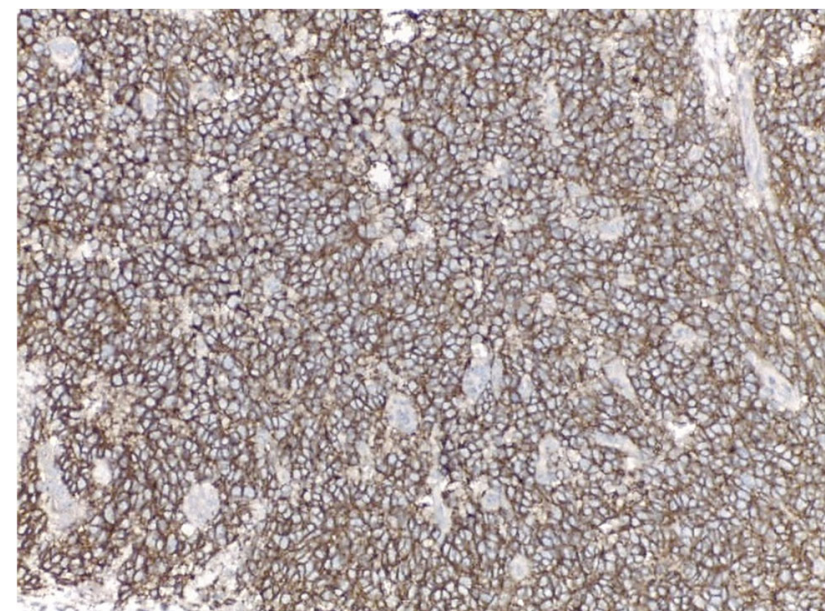

Figure 4 - Diffuse tumor cell immunoreactivity for CD99 (IHC with DAB chromogen, 200×). CD99: Cluster of differentiation 99; DAB: 3,3'-Diaminobenzidine; IHC: Immunohistochemistry.

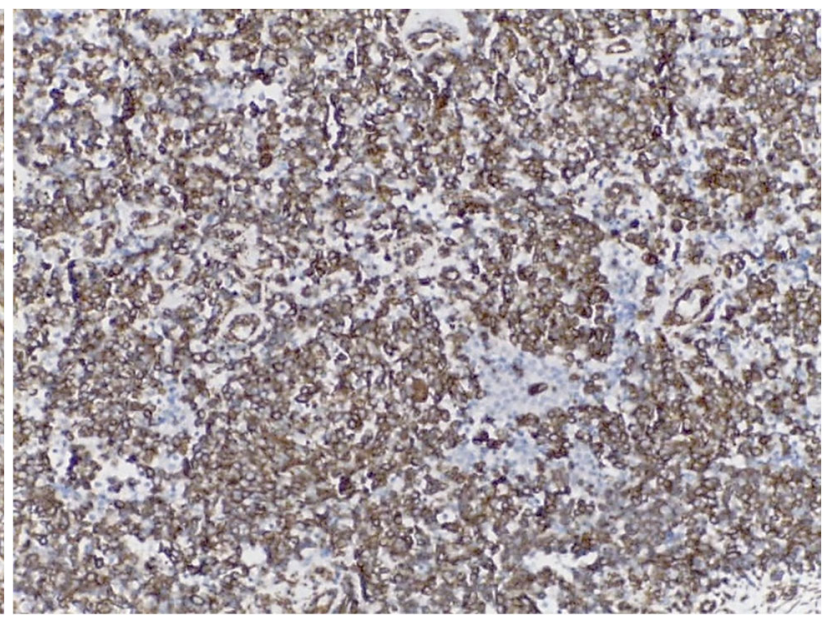

Figure 5 - Diffuse tumor cell immunoreactivity for vimentin (IHC with $\mathrm{DAB}$ chromogen, 200×).

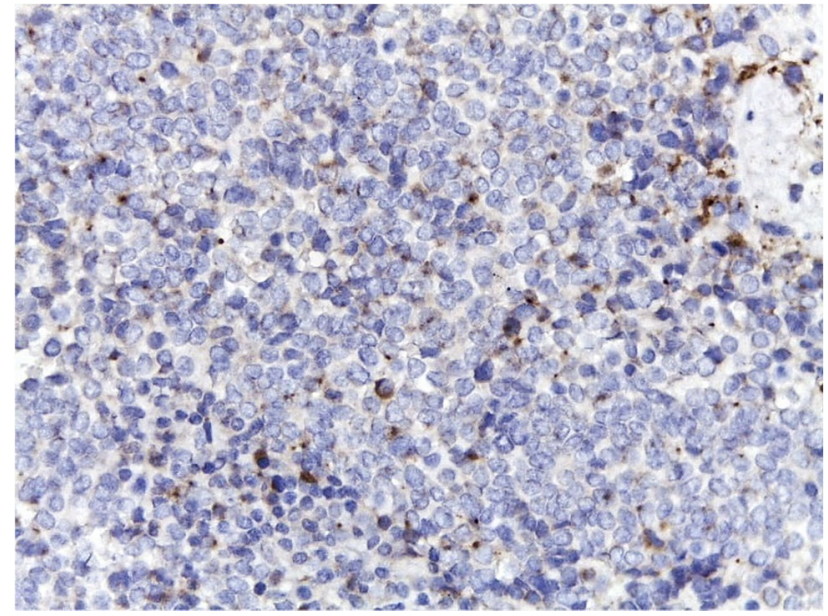

Figure 6 - Focal tumor cell immunoreactivity for synaptophysin (IHC with DAB chromogen, 400×).

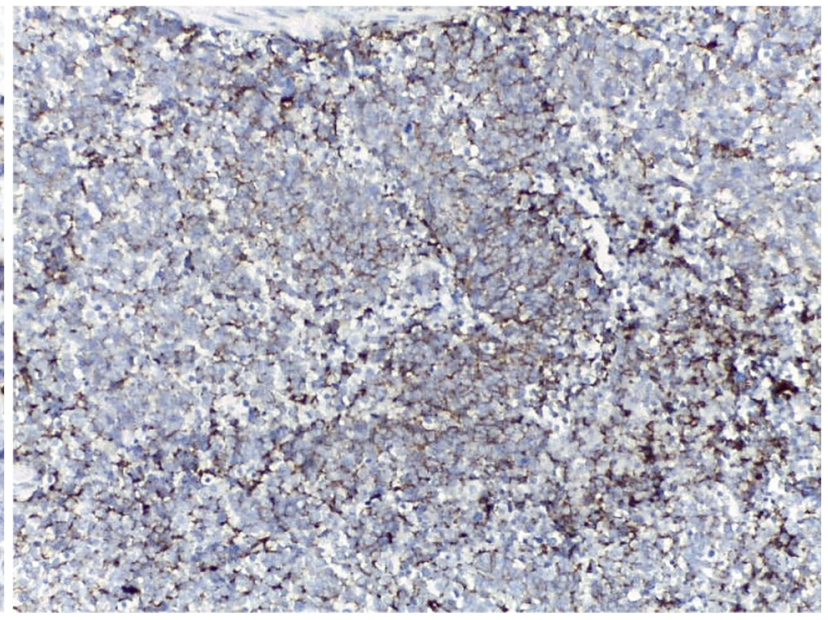

Figure 7 - Focal tumor cell immunoreactivity for CD56 (IHC with DAB chromogen, 200×). CD56: Cluster of differentiation 56.

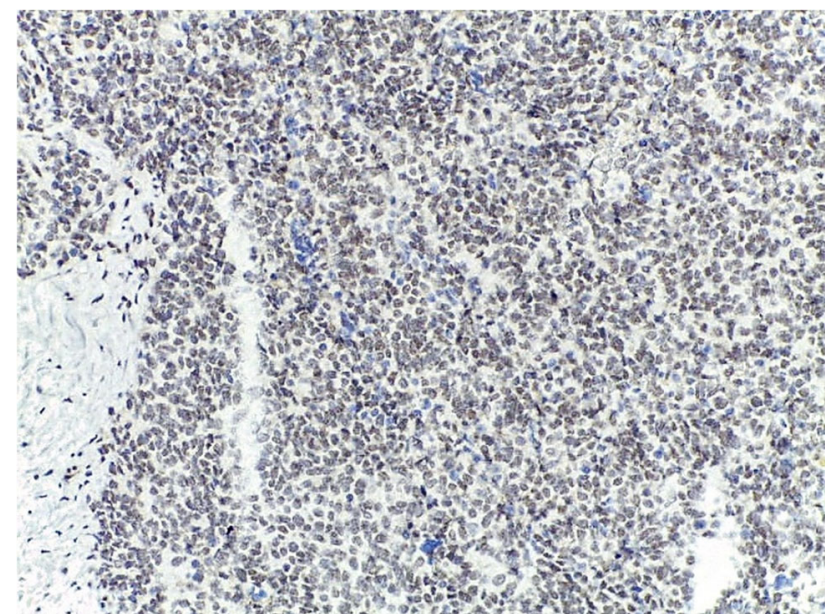

Figure 8 - Diffuse tumor cell immunoreactivity for FLI1 (IHC with DAB chromogen, 200×). FLI1: Friend leukemia integration 1.

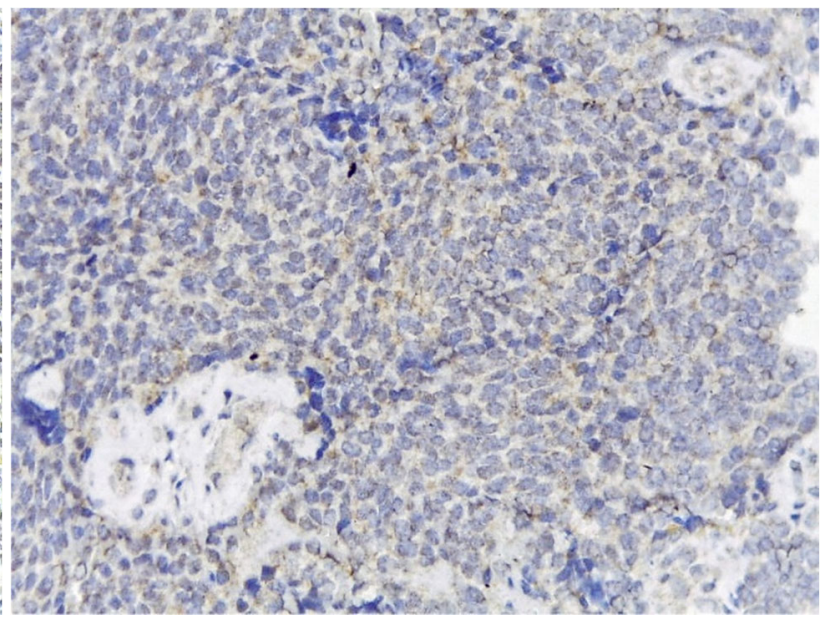

Figure 9 - Weak tumor cell immunoreactivity for GFAP (IHC with DAB chromogen, $400 \times$ ). GFAP: Glial fibrillary acidic protein. 
At three-month follow-up, native and intravenous contrast pelvic MRI revealed a newly formed intrapelvic mass, with heterogeneous structure, mainly cystic, with multiloculated, hypodense areas and variable septa, located in the right adnexal region, measuring approximately $93 / 75 / 60 \mathrm{~mm}$. The tumor had contact with the rectum, ileal loops, uterus, and posterior wall of the bladder, without signs of invasion. The tumor had a posterior tissue component that extended perirectally to the right, in touch with the mesorectal fascia. A small hemorrhagic collection in the bottom of the Douglas pouch was observed in contact with the intrapelvic mass. No suspected intra-abdominalpelvic lymphadenopathy was observed. The uterus had normal size, without suspicious lesions detectable within the body or the cervix. Due to the infiltrative character of the tumor, no second-look intervention has been performed. The case has been thoroughly analyzed in a multidisciplinary team (MDT), which included an oncologist, a gynecologist, and a pathologist, where they concluded that the best approach would be combined radiation therapy and chemotherapy. The chemotherapy scheme included the following medication: Doxorubicin, Cyclophosphamide, Vincristine and Actinomycin. The radiation therapy aims at shrinking the dimensions of the tumor, which would make possible a new surgical intervention. The patient is currently under adjuvant treatment and has monthly appointments planned with the oncologist, which also include imagistic surveillance. So far, after six months, the course has been uneventful and imaging surveillance shows partial tumor regression. Although promising studies have reported that EWSR1-FLI1 antagonists stop the in vitro proliferation rate of tumors included in the Ewing family of tumors, there are no approved therapies available for human patients with tumors harboring this mutation [11].

\section{ㅁ Discussions}

From a clinical point of view, our case confirms the usual presentation of ovarian PNETs, in which an abdominal mass or abdominal pain represent the most common symptoms [4, 12-14]. However, the patient also presented with abnormal vaginal bleeding, which is an uncommon presentation that has been reported only once in the scientific literature [14]. The patient age (30 years) respects the characteristic pattern of age distribution encountered in patients with primary ovarian PNET [4, 15, 16]. A maximum size of $110 \mathrm{~mm}$ has also been reported in other scientific articles [3, 12], although the dimensions reported in the literature vary from $6.5 \mathrm{~cm}$ to $16.5 \mathrm{~cm}$ $[5,17]$.

Grossly, the tumor from our case had an inhomogeneous appearance, with a multilocular cystic structure and solid areas featuring extensive necrosis. In comparison to the scientific literature, a cystic component was more commonly described in the differentiated variant of cPNET [4] and a multilocular cystic component was present in $85.7 \%$ of all cystic PNETs [15]. Nili et al. have also reported the presence of large areas of necrosis in pPNETs [18].

From a microscopic point of view, ovarian PNETs can be divided into cPNETs and pPNETs. The former can be further subdivided into three variants: differentiated, anaplastic and primitive [4]. cPNETs arise in the presence of central nervous tissue, and are thought to stem from a teratoma, although scientific articles have described the conjunction between cPNET and endometrioid carcinoma or mixed malignant mesodermal tumor [3, 19]. The differentiated variant of cPNET can resemble either an ependymoma or a neurocytoma, an oligodendroglioma or an astrocytoma [20-24]. The primitive variant of cPNET, as the name implies, features the most primitive histological aspect of a neuroectodermal tumor and frequently resembles a medulloblastoma, neuroblastoma, medulloepithelioma or ependymoblastoma [3]. This variant is frequently associated with a teratoma [7]. The anaplastic variant of cPNET features large areas of necrosis and microvascular proliferations within a high-grade glioma, aspect characteristic for a glioblastoma [25].

IHC studies revealed a CD99-negative proliferation of small cells, that also exhibits immunoreactivity for neuroendocrine markers, especially CD56 and synaptophysin $[6,7]$. According to the scientific literature, chromogranin immunoexpression is not a classical feature of these tumors $[3,10]$. Correlating to their origin in the nervous tissue, one can also observe diffuse immunoreactivity towards GFAP, which is not a characteristic feature of the pPNETs [3].

pPNETs are rarer than cPNETs and they are composed of a diffuse proliferation of small blue cells, without resembling any specific tumor. They are characterized by tapered cytoplasmic processes, without intracytoplasmic glycogen vesicles [10]. However, they can frequently feature rosettes of Homer Wright type or FlexnerWintersteiner type, as did the case presented above [10]. McCluggage also noted the presence of variable areas of necrosis, fibrosis, and mitotic activity [26]. From an IHC point of view, pPNET features diffuse immunoexpression of CD99 (MIC2 protein), as was observed in our case. Classical IHC phenotype includes immunoreactivity for CD56, synaptophysin, and also nuclear positivity for FLI1 and for NK2 homeobox 2 (NKX2.2) $[10,26]$. The first three immunomarkers were also positive in the above presented case, in consonance with the scientific literature. Other IHC markers that can be occasionally expressed in pPNET are neuron-specific enolase (NSE), S100 protein, oligodendrocyte transcription factor 2 (OLIG2) and cluster of differentiation 57 (CD57/ Leu7) $[10,24]$. Although some authors have reported that pan-CK AE1/AE3 can be either negative or show patchy positivity, in our case, no immunoreactivity towards this marker was noticed in the tumoral cells $[10,26]$. The Ki67 proliferative index showed a value of $60 \%$, being is in the upper spectrum of the values reported in the literature, which varies between $3 \%$ and $90 \%[6,20]$.

Classical $\mathrm{t}(11 ; 22)(\mathrm{q} 24 ; \mathrm{q} 12)$ translocation, characteristic of the ESWR-FLI1 fusion, which was also confirmed in our case, has been previously reported in multiple scientific articles [8-10]. Additionally, gene amplification of epidermal growth factor receptor $(E G F R)$ and deletions of the retinoblastoma $(R B)$ gene have also been reported [26]. One should also be aware of the translocation variant $\mathrm{t}(21 ; 22)(\mathrm{q} 22$.q12), which can only be identified by fluorescence in situ hybridization (FISH) analysis, due 
to omission of this translocation upon polymerase chain reaction (PCR) testing [9, 10]. Losses of chromosomes $1 \mathrm{p}, 1 \mathrm{q}, 6 \mathrm{p}, 6 \mathrm{q}, 4 \mathrm{q}, 7 \mathrm{q}$ and $13 \mathrm{q}$, as well as deletions of Xq, 7p, 2p 1q, 18q, 9q have also been documented in a thorough analysis [27].

Although ovarian PNETs are extremely rare tumors, which do not have a well-defined "golden standard" for treatment, the usual therapeutic management involves total hysterectomy with bilateral salpingo-oophorectomy, followed by adjuvant chemotherapy and radiotherapy $[3-5,10,26]$. However, there have been cases reported in which the patient wanted to conserve her fertility and/or who opted only for cystectomy or for surgical excision of the tumor followed by chemotherapy $[6,12$, 13]. A similar situation was also present in our case, where the patient endured only tumorectomy, followed by adjuvant chemotherapy and radiotherapy. Demirtas et al. have even reported a case of primary ovarian PNET where the patient successfully delivered two babies and that had a follow-up of more than three years without any recurrences [12]. Nonetheless, in our case, the close follow-up revealed a subsequent recurrence that developed in less than three months from the surgical intervention. Ostwal et al. have also reported a primary ovarian PNET which developed a recurrence after one and a half year and who had a fatal outcome soon after, although the standard treatment was followed (total hysterectomy followed by chemotherapy) [9]. Regarding the fertility potential of patients with ovarian PNET, noteworthy is also the case reported by Lim et al., in which the tumor was discovered as an incidental finding during a pregnancy [6].

The most important differential diagnoses with extremely different therapeutic management and patient outcome are granulosa cell tumors and germ cell tumors.

\section{ㅁ Conclusions}

Due to the rarity of this entity, the clinical, histopathological, and prognostic implications of ovarian PNETs are still unclear. There are no standard therapeutic guidelines, and an individualized strategy is currently the best practice. In our experience, tailored combined chemotherapy and radiation therapy appears to be the best choice for these types of tumors, especially in advanced stages.

\section{Conflict of interests}

The authors declare that they have no conflict of interests.

\section{References}

[1] Hart MN, Earle KM. Primitive neuroectodermal tumors of the brain in children. Cancer, 1973, 32(4):890-897. https://doi.org/ 10.1002/1097-0142(197310)32:4<890::aid-cncr2820320421 >3.0.co;2-o PMID: 4751919

[2] Trancău IO. Chromosomal translocations highlighted in primitive neuroectodermal tumors (PNET) and Ewing sarcoma. J Med Life, 2014, 7(Spec Iss 3):44-50. PMID: 25870694 PMCID: PMC4391400

[3] Nucci MR, Oliva E. Diagnostic pathology: gynecological. $2^{\text {nd }}$ edition, Elsevier, Salt Lake City, USA, 2018, 464-468. https:// www.elsevier.com/books/diagnostic-pathology-gynecological/ nucci/978-0-323-54815-1

[4] Kleinman GM, Young RH, Scully RE. Primary neuroectodermal tumors of the ovary. A report of 25 cases. Am J Surg Pathol, 1993, 17(8):764-778. https://doi.org/10.1097/00000478-199 308000-00002 PMID: 8393302
[5] Fischer G, Odunsi K, Lele S, Mhawech P. Ovarian primary primitive neurectodermal tumor coexisting with endometrioid adenocarcinoma: a case report. Int J Gynecol Pathol, 2006, 25(2):151-154. https://doi.org/10.1097/01.pgp.0000185408. 31427.4e PMID: 16633064

[6] Lim YK, Ku CW, Teo GC, Lim SL, Tee CS. Central primary primitive neuroectodermal tumor (cPNET) arising from an ovarian mature cystic teratoma in pregnancy: a case report and review of medical literature. Gynecol Oncol Case Rep, 2013, 4:56-59. https://doi.org/10.1016/j.gynor.2013.01.004 PMID: 24371678 PMCID: PMC3862304

[7] Herrington CS (ed), World Health Organization (WHO) Classification of Tumours Editorial Board. Female genital tumours. $5^{\text {th }}$ edition, vol. 4, International Agency for Research on Cancer (IARC) Press, Lyon, France, 2020, 136. https://publications. iarc.fr/Book-And-Report-Series/Who-Classification-Of-Tumours/ Female-Genital-Tumours-2020

[8] Lin PP, Brody RI, Hamelin AC, Bradner JE, Healey JH, Ladanyi M. Differential transactivation by alternative EWSFLI1 fusion proteins correlates with clinical heterogeneity in Ewing's sarcoma. Cancer Res, 1999, 59(7):1428-1432. PMID: 10197607

[9] Ostwal V, Rekhi B, Noronha V, Basak R, Desai SB, Maheshwari A, Prabhash K. Primitive neuroectodermal tumor of ovary in a young lady, confirmed with molecular and cytogenetic results - a rare case report with a diagnostic and therapeutic challenge. Pathol Oncol Res, 2012, 18(4):11011106. https://doi.org/10.1007/s12253-012-9503-2 PMID: 22311546

[10] Kurman RJ, Hedrick Ellenson L, Ronnett BM (eds). Blaustein's pathology of the female genital tract. $7^{\text {th }}$ edition, Springer Nature Switzerland AG, 2019, 1451-1453. https://doi.org/ 10.1007/978-3-319-46334-6

[11] Mateo-Lozano S, Gokhale PC, Soldatenkov VA, Dritschilo A, Tirado OM, Notario V. Combined transcriptional and translational targeting of EWS/FLI-1 in Ewing's sarcoma. Clin Cancer Res, 2006, 12(22):6781-6790. https://doi.org/10.1158/1078-0432. CCR-06-0609 PMID: 17121899

[12] Demirtas E, Guven S, Guvendag Guven ES, Baykal C, Ayhan A. Two successful spontaneous pregnancies in a patient with a primary primitive neuroectodermal tumor of the ovary. Fertil Steril, 2004, 81(3):679-681. https://doi.org/10.1016/j. fertnstert.2003.08.036 PMID: 15037420

[13] Jaramillo-Huff A, Bakkar R, McKee JQ, Sokkary N. Primary primitive neuroectodermal tumor arising from an ovarian mature cystic teratoma in a 12-year-old girl: a case report. J Pediatr Adolesc Gynecol, 2017, 30(4):511-512. https://doi.org/10. 1016/j.jpag.2016.11.007 PMID: 27919713

[14] Georgescu TA, Bohiltea R, Munteanu O, Grigoriu C, Paunica I, Sajin M. A mini-review regarding the carcinogenesis and morphology of serous tumors of the ovary, Fallopian tube and peritoneum. J Mind Med Sci, 2021, 8(1):44-52. https://doi.org/ 10.22543/7674.81.p4452

[15] Chiang S, Snuderl M, Kojiro-Sanada S, Quer Pi-Sunyer A, Daya $D$, Hayashi T, Bosincu L, Ogawa F, Rosenberg AE, Horn LC, Wang L, lafrate AJ, Oliva E. Primitive neuroectodermal tumors of the female genital tract: a morphologic, immunohistochemical, and molecular study of 19 cases. Am J Surg Pathol, 2017, 41(6):761-772. https://doi.org/10.1097/ PAS.0000000000000831 PMID: 28296680 PMCID: PMC 5525138

[16] Anfinan NM, Sait KH, Al-Maghrabi JA. Primitive neuroectodermal tumor of the ovary. Saudi Med J, 2008, 29(3):444-446. PMID: 18327377

[17] Chu LH, Chang WC, Kuo KT, Sheu BC. Primary primitive neuroectodermal tumor of the ovary. Taiwan J Obstet Gynecol, 2014, 53(3):409-412. https://doi.org/10.1016/j.tjog.2013.08. 005 PMID: 25286803

[18] Nili F, Sedighi Moghadam Pour A, Moradi Tabriz H, Sedighi Moghadam Pour P, Saffar H. Peripheral primitive neuroectodermal tumor of the ovary: the report of two rare cases. Iran J Pathol, 2018, 13(4):467-470. PMID: 30774687 PMCID: PMC6358556

[19] Mott RT, Murphy BA, Geisinger KR. Ovarian malignant mixed mesodermal tumor with neuroectodermal differentiation: a multifaceted evaluation. Int J Gynecol Pathol, 2010, 29(3): 234-238. https://doi.org/10.1097/PGP.0b013e3181bd413c PMID: 20407321 
[20] Opris I, Ducrotoy V, Bossut J, Lamy A, Sabourin JC. Oligodendroglioma arising in an ovarian mature cystic teratoma. Int J Gynecol Pathol, 2009, 28(4):367-371. https://doi.org/ 10.1097/PGP.0b013e318196c4c0 PMID: 19483626

[21] Yoder N, Marks A, Hui P, Litkouhi B, Cron J. Low-grade astrocytoma within a mature cystic teratoma in an adolescent patient. J Pediatr Adolesc Gynecol, 2018, 31(3):325-327. https://doi.org/10.1016/j.jpag.2017.10.005 PMID: 29107097

[22] Hirschowitz L, Ansari A, Cahill DJ, Bamford DS, Love S. Central neurocytoma arising within a mature cystic teratoma of the ovary. Int J Gynecol Pathol, 1997, 16(2):176-179. https://doi.org/10.1097/00004347-199704000-00016 PMID: 9100074

[23] Berceanu C, Cîrstoiu M, Mehedinţu C, Brătilă P, Berceanu S, Vlădăreanu $S$, Bohîlţea R, Brătilă E. Hormone deficiency and its impact on the lower urinary tract. Proceedings of the $13^{\text {th }}$ National Congress of Urogynecology (UROGYN 2016), Filodiritto Editore, Bologna, Italy, 2016, 29-38. https://www. filodiritto.com/proceedings?field_cat_proc $=\&$ field_cat_proce edings_target_id=All\&page $=5$

[24] Murdock T, Orr B, Allen S, Ibrahim J, Sharma R, Ronnett BM, Rodriguez FJ. Central nervous system-type neuroepithelial tumors and tumor-like proliferations developing in the gynecologic tract and pelvis: clinicopathologic analysis of 23 cases. Am J Surg Pathol, 2018, 42(11):1429-1444. https:// doi.org/10.1097/PAS.0000000000001131 PMID: 30074494

[25] Louis DN, Perry A, Wesseling P, Brat DJ, Cree IA, FigarellaBranger D, Hawkins C, Ng HK, Pfister SM, Reifenberger G, Soffietti R, von Deimling A, Ellison DW. The 2021 WHO Classification of Tumors of the Central Nervous System: a summary. Neuro Oncol, 2021, 23(8):1231-1251. https://doi.org/ 10.1093/neuonc/noab106 PMID: 34185076 PMCID: PMC 8328013

[26] McCluggage WG. Ovarian neoplasms composed of small round cells: a review. Adv Anat Pathol, 2004, 11(6):288-296. https://doi.org/10.1097/01.pap.0000138146.357376.1e PMID: 15505529

[27] Chow SN, Lin MC, Shen J, Wang S, Jong YJ, Chien CH. Analysis of chromosome abnormalities by comparative genomic hybridization in malignant peripheral primitive neuroectodermal tumor of the ovary. Gynecol Oncol, 2004, 92(3): 752-760. https://doi.org/10.1016/j.ygyno.2003.11.027 PMID: 14984937

\section{Corresponding authors}

Antonia Carmen Lisievici, Assistant Professor, MD, PhD, Department of Pathology, Carol Davila University of Medicine and Pharmacy, 8 Eroilor Sanitari Avenue, Sector 5, 050474 Bucharest, Romania; Phone +40741-339 830, e-mail: lisievici.carmen@yahoo.com

Tiberiu Augustin Georgescu, Assistant Professor, MD, PhD, Department of Pathology, Carol Davila University of Medicine and Pharmacy, 8 Eroilor Sanitari Avenue, Sector 5, 050474 Bucharest, Romania; Phone +40721-468 622, e-mail: tiberiuaugustin.georgescu@gmail.com

Received: July 30, 2021

Accepted: December 12, 2021 